\title{
A META-ANALYSIS OF MOTIVATIONAL INTERVIEWING INTERVENTIONS FOR PEDIATRIC HEALTH BEHAVIOR CHANGE
}

\section{BY}

\section{LAURIE A. GAYES}

Submitted to the graduate degree program in Clinical Child Psychology Program and the Graduate Faculty of the University of Kansas in partial fulfillment of the requirements for the Master of Arts.

Chairperson Ric G. Steele, Ph.D., ABPP

Michael C. Roberts, Ph.D., ABPP

Paula Fite, Ph.D.

Date Defended: April 30, 2013 
The Thesis Committee for LAURIE A. GAYES

Certifies that this is the approved version of the following thesis:

\section{A META-ANALYSIS OF MOTIVATIONAL INTERVIEWING INTERVENTIONS FOR PEDIATRIC HEALTH BEHAVIOR CHANGE}

Chairperson Ric G. Steele, Ph.D., ABPP

Date approved: April 30, 2013 


\begin{abstract}
Motivational interviewing (MI) is an empirically supported intervention that has shown effectiveness in moving people towards positive lifestyle choices. Although originally designed for adult substance users, MI has since expanded to other health concerns with a range of clients age groups. The following meta-analysis analyzed MI's effectiveness in eliciting behavior change in pediatric health domains. Thirty-seven empirical studies were included in this metaanalysis, encompassing eight health domains. The overall effect size (Hedge's $g$ ) in this population as compared to both other active treatments and no treatment was $0.282(95 \% \mathrm{CI}$ [0.242-0.323]), slightly higher than a "small" effect size, and also slightly higher than what has been typically found in the substance literature. The effectiveness of MI in pediatric domains was moderated by factors such as practitioner background, health domain, and the family member who participated. Effect sizes varied by health condition such that the health domains with the largest overall effect sizes were Type 1 Diabetes, asthma, and calcium intake. MI seems to be most effective when both parent and child participate in sessions and when the cultural background of the practitioner matches the family. Unexpectedly, number of MI sessions and follow-up length were not significant moderators. Overall, these findings indicate that MI is an effective and appropriate intervention for targeting child health behavior changes.
\end{abstract}




\section{Table of Contents}

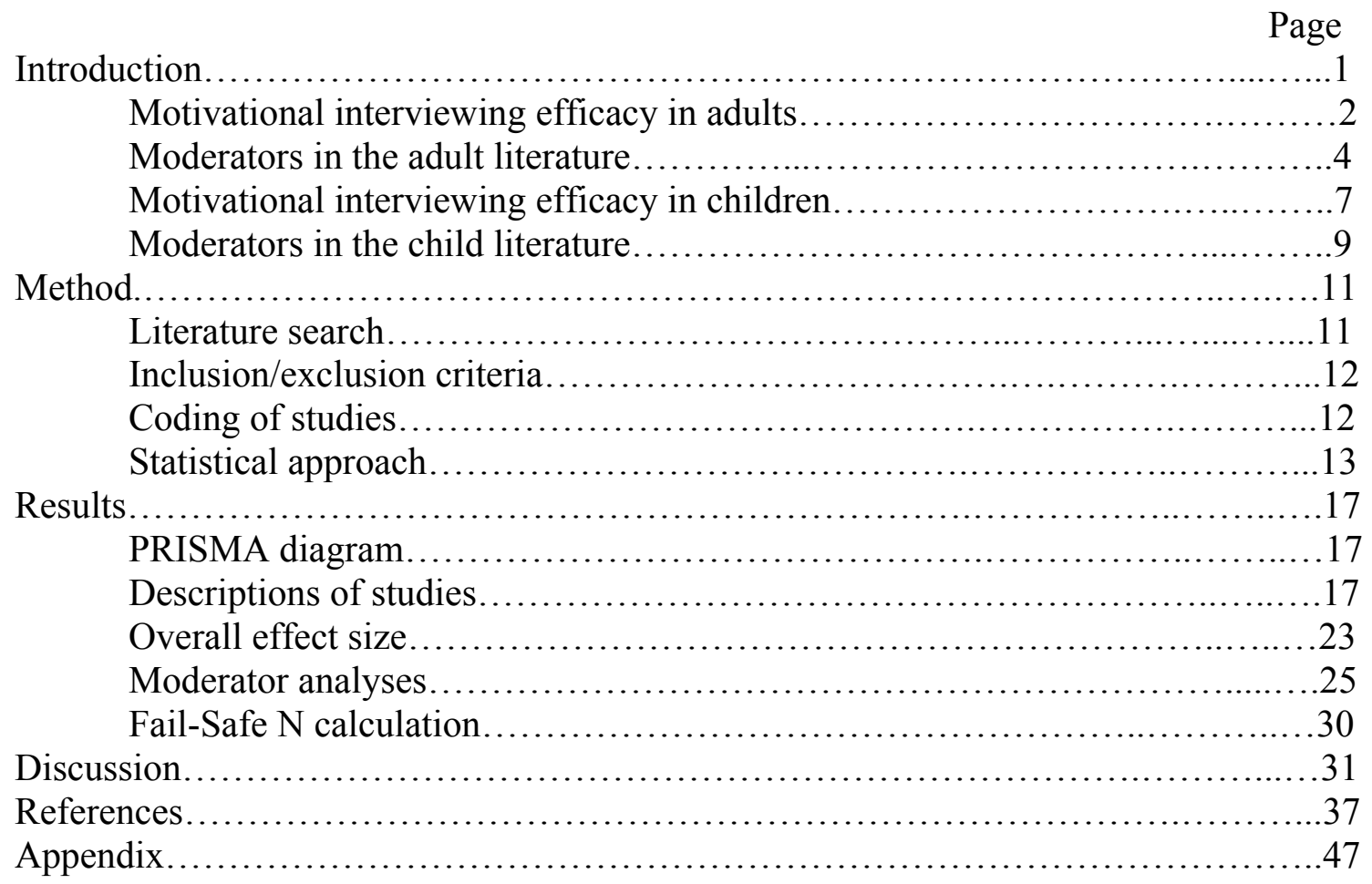




\section{Introduction}

Motivational interviewing (MI) is an empirically supported intervention that has shown promise in improving a range of health outcomes in a relatively brief amount of time (Lundahl, Kunz, Brownell, Tollefson, \& Burke, 2010). MI is a clinical style of patient-professional interaction that enhances the patient's intrinsic motivation for behavior change. MI has been described by its founders as being "a client-centered, directive method for enhancing intrinsic motivation to change by exploring and resolving ambivalence" (Miller \& Rollnick, 2002, p. 25). MI was developed by Miller and Rollnick in the 1980s as an alternative to traditional treatments for substance abuse in adults (Miller \& Rose, 2009). Over the last 30 years, MI has become an established treatment for adult substance abuse and has emerged as a promising method for treating a variety of behaviorally-based health conditions and for a range of age groups (Miller \& Rollnick, 2002).

MI is thought to work by incorporating four basic techniques into interactions between the therapist and the client: expressing empathy, developing discrepancy, rolling with resistance, and supporting self-efficacy (Miller \& Rollnick, 2002). These four techniques depend on the therapist's ability to foster a warm empathetic relationship with the client in which the client can freely express ambivalence for change and the role the problematic behavior plays in his/her life (Miller \& Rollnick, 2002). Therapists assist clients in developing a discrepancy between the realities of their current problematic behavior and their values and future goals. This discrepancy prompts cognitive dissonance within the client, who must reconcile his/her current lifestyle with those consistent with their values and goals. Only after the client has expressed motivation for behavior change is there a discussion of how the client might enact behavior change. Unlike traditional behavior change strategies in which there is an assumption that the 
client is already committed to change (White \& Miller, 2007), MI assumes that there is a degree of ambivalence in the client towards change and the client is supported by the therapist in reflecting on the challenges associated with behavior change (Miller \& Rose, 2009). Finally, the client's personal strengths and tools for making change are highlighted and their confidence in ability to change is encouraged.

\section{Motivational Interviewing Efficacy in Adults}

Several recent meta-analyses have examined the efficacy of MI in treating a range of outcomes in adults. Most of the meta-analyses focused on studies targeting substance abuse, with a smaller number of studies targeting physical and mental health outcomes (Burke, Arkowitz, \& Menchola, 2003; Burke, Dunn, Atkins, \& Phelps, 2004; Hettema, Steele, \& Miller, 2005; Lundahl et al., 2010; Rubak, Sandbaek, Lauritzen, \& Christensen, 2005). Several metaanalyses solely targeted alcohol abuse (Carey, Scott-Sheldon, Carey, \& DeMartini, 2007; Vasilaki, Hosier, \& Cox, 2006) or nicotine addiction (Heckman, Egleston, \& Hoffman, 2010; Hettema \& Hendricks, 2010), and one dissertation solely targeted obesity treatment (DiRosa, 2009).

Overall results indicated that MI yields small to medium effect sizes across all outcome conditions. The largest and most comprehensive meta-analysis to date found an overall average effect size $(g)$ of 0.22 , and reported that the range of effect sizes $(g)$ for individual studies extended from -1.40 to 2.06 (Lundahl et al., 2010). Other meta-analyses yielded similar results, with Burke and colleagues (2003) reporting small to medium effect sizes ( $d=.25$ to .57$)$, and Vasilaki (2006) reporting effect sizes ranging from $d=.18$ to .43. Burke and colleagues (2004) reported a slightly higher range of effect sizes from $d=.35$ to .56 . Several systematic reviews 
that did not calculate effect sizes reported instead that a majority of studies reviewed yielded positive outcomes (e.g., Dunn, Deroo, \& Rivara, 2002; Rubak et al., 2005).

Specifically within the substance abuse domain, effect sizes consistently ranged from small to medium. For alcohol treatment, numerous researchers reported overall effect sizes in the small range (i.e., $d=0.11$ to 0.26 ) (Carey et al., 2007; Hettema et al., 2005; Lundahl et al., 2010; Vasilaki et al., 2006), while Burke $(2003,2004)$ reported small to medium effect sizes $(d=$ 0.35 to 0.09$)$ for change in drinking frequency and medium effect sizes $(d=0.53)$ for change in level of intoxication. For marijuana treatment, Lundahl (2010) reported a small average effect size of $d=0.26$. For tobacco problems, effect sizes ranged from $d=0.14$ to 0.25 (Hettema \& Hendricks, 2010; Hettema et al., 2005; Lundahl et al., 2010). For other drug problems, effect sizes ranged from $d=0.08$ (Lundahl et al., 2010) to $d=0.29$ (Hettema et al., 2005). No metaanalysis found in this search reported a combined effect size solely for substance use interventions, but it would likely be in the small to medium range.

Outside of substance abuse treatment, MI has been implemented across a range of health behaviors both alone or in conjunction with other treatments to increase physical health in areas such as adherence to medication and medical regimen; weight loss; exercise; eating disorder treatment; safe water drinking practices; oral health; and fruit and vegetable intake (Lundahl et al., 2010; Van Dorsten, 2007). Using MI in these health domains tends to yield similar or slightly higher effect sizes than for substance abuse treatments, although the results appear to be highly variable. Hettema and colleagues (2005) found studies using MI to treat physical health behaviors in adults yielded negative (eating disorders; average $d=-0.07$ ) to large (diet and exercise; average $d=0.78$ ). Lundahl and colleagues (2010) reported small average effect sizes for studies using MI to increase healthy behavior (average $d=0.21$ ), small average effect sizes 
for reducing risky behavior (e.g., unprotected sex) (average $d=0.14$ ), and small average effect sizes for reducing symptoms of eating disorders (average $d=0.18$ ). Similarly, a meta-analytic dissertation by DiRosa (2009) on MI for obesity treatment yielded small to medium weighted effect sizes for MI in dietary intake change (average $d=0.35$ ), change (increase) in physical activity (average $d=0.24$ ), and weight loss (average $d=0.19$ ). The combined weighted effect size for these health outcomes studies was small $(d=0.29)$, consistent with the substance abuse literature (DiRosa, 2009).

MI interventions on health behaviors have demonstrated positive effects on secondary outcomes variables as well. A systematic review of eight studies in the fields of diabetes, asthma, hypertension, hyperlipidemia, and heart disease found that the majority of studies yielded positive outcomes on psychological, physiological, and lifestyle areas (Knight, McGowan, Dickens, \& Bundy, 2006). Burke and colleagues (2003) found that over all treatment populations, MI treatments yield medium effect sizes (average $d=0.47$ ) for measures of social impact, such as social, occupational, and physical problems related to the target symptom (e.g., absence from work or school, memory loss, legal problems).

\section{Moderators in Adult Literature}

Numerous meta-analyses indicate that results have varied (i.e., were moderated) by comparison group, target health domain, dosage of treatment, treatment characteristics, participant characteristics, and practitioner characteristics, although the direction of the effect was not always consistent (Dunn et al., 2001; Burke et al., 2003; Hettema \& Hendericks, 2010; Lundahl et al., 2010; Rubak et al., 2005; Vasilaki et al., 2006). Studies comparing MI to weak comparison groups (no treatment group or minimal treatment group) demonstrated effect sizes in the small to medium range (average $g=0.28$ ) and those studies that compared MI to another 
active another treatment group found small or non-significant effect sizes (average $g=0.09$ ); (Lundahl et al., 2010). MI seems to yield favorable outcomes across all health domains studied, but results on identifying particularly effective or ineffective domains are mixed. For example, Lundahl (2010) reported largest effect sizes for smoking cessation and gambling interventions and smallest effect sizes for change in emotional well-being and eating disorder interventions. In contrast, Dunn and colleagues (2001) reported larger effect sizes in diet and exercise studies and smaller among MI interventions to affect smoking cessation. Likewise, there appears to be a dose impact of MI, where the effect size increased with more sessions (Burke et al., 2003; Lundahl et al., 2010; Rubak et al., 2005; Vasilaki et al., 2006); although Hettema (2010) found that briefer sessions (less than one hour) delivered a more long-term impact than longer sessions.

Treatment and study characteristics also moderate outcomes. Lundahl (2010) found that effect sizes were greater when a manual was not used than when it was used and, when paired with another treatment, that MI was most effective when placed first. High quality studies (measured across several study characteristics) yielded smaller effect sizes than low quality studies (Burke et al., 2003; Lundahl et al., 2010), perhaps suggesting that the true effect of MI is small but significant. Two meta-analyses reported no differences in effect sizes when MI was compared to other active treatments over follow-up periods (Heckman et al., 2010; Lundahl et al., 2010). However, one meta-analysis found that for smoking cessation studies, MI was equivalent to other active treatments in the short term, but yielded more lasting effects in the long term (Hettema \& Hendricks, 2010). Another meta-analysis found that for drinking reduction studies, the effect of MI peaked at 3 months post treatment and behavior change was no longer significant at 6 months post treatment (Vasilaki et al., 2006). 
Participant characteristics, such as age and ethnicity, also appear to moderate the impact of MI, although the direction is not consistent. Older participants tend to demonstrate better outcomes than younger participants, but only when MI was compared to treatment as usual (slope $=0.006, p \leq .01)$ (Lundahl et al., 2010). In contrast, Hettema and Hendricks (2010) found that studies with adolescent samples (under age 18) had significant combined effect sizes at both short term and long term follow up points $(d=0.15$ and $d=0.11)$, while in adult samples, the effect sizes were not significant. Participants of minority ethnic/racial groups (Hettema et al., 2005; Lundahl et al., 2010) and international participants (Hettema \& Hendricks, 2010) demonstrate larger effect sizes than American Caucasian participants, but the data on MI with minority groups are still limited. MI may also be more effective with clients who are angry, resistant, and less ready to change than clients who already want to change (Hettema et al., 2005). MI seems to be effective for both treatment-seeking and non-treatment-seeking samples (Vasilaki et al., 2006) and gender has not been found to be a significant moderator (Hettema \& Hendricks, 2010; Vasilaki et al., 2006).

In terms of practitioner characteristics, data suggest that profession and education level are not related to outcomes (Lundahl et al., 2010; Heckman et al., 2010), but that the therapist's display of empathy and the quality of the therapeutic relationship are significantly related (Miller $\&$ Baca, 1983).

In summary, the current state of the literature suggests that MI demonstrates comparable outcomes to traditional treatments in supporting a wide range of behavioral changes, although it is most well supported in substance use interventions. MI demonstrates an overall effect size in the small to medium range, consistent with other active treatments (i.e., not treatment as usual). Some variation in results can be attributed to several moderating factors, including type of 
comparison group, health domain, study characteristics, participant characteristics, and practitioner characteristics. However, it has also been suggested that MI may offer several advantages to traditional active treatments, including facilitating changes in less time and fewer sessions, being economically feasible to deliver, offering fewer opportunities for attrition, and allowing a wide range of providers to deliver treatment (Butler, Rollnick, Cohen, Russel, Bachmann, \& Stott, 1999; Lundahl et al., 2010; Rubak et al., 2005; Vasilaki et al., 2006).

\section{Motivational Interviewing Efficacy in Children}

While the literature on MI in adult populations is robust, the evidence base for MI as an appropriate and effective intervention with children and adolescents is only emerging. As in the adult literature, the majority of work has been conducted in the substance abuse area. Several systematic reviews have suggested that MI is effective with adolescent substance abusers by accelerating the natural maturation process of reduced drinking and drug-using behavior from adolescence to adulthood (Tevyaw \& Monti, 2004; Wachtel \& Staniford, 2010). As a subset for a larger analysis of brief interventions for substance-using adolescents, Tait and Hulse (2003) analyzed four interventions using the MI approach and reported a small but significant aggregate effect size $(d=0.24)$, which was slightly larger than the overall effect size for all brief interventions $(d=0.13)$. However, to date, only one rigorous meta-analysis has been conducted on interventions with MI for children and adolescents. Jensen and colleagues (2011) analyzed

21 studies of interventions for adolescent substance use across tobacco, alcohol, marijuana, and illicit drug use. These researchers reported a small but significant mean effect size $(d=0.17)$ that persisted over time, consistent with what has been found in the adult literature (Carey et al., 2007; Hettema et al., 2005; Lundahl et al., 2010, Vasilaki et al., 2006). 
In addition to their quantitative summary of effect sizes, Jensen and colleagues (2011) provided some of the first comprehensive descriptions of how MI has been administered to youth. They reported that the majority of studies involved only one session of MI, although the mean number of intervention sessions was four. Follow-up periods ranged from one month to 24 months. MI was most often the sole treatment, but was also frequently combined with cognitivebehavioral therapy. Perhaps reflecting a high number of referrals for substance abuse treatment (Battjes, Gordon, O’Grady, \& Kinlock, 2004), male participants slightly outnumbered female participants and white participants made up the largest proportion of the treatment population. Interventionists had a wide variety of backgrounds and education levels, and few studies reported treatment fidelity or counselor training.

Despite the fact that MI requires a somewhat sophisticated level of insight and discussion of mature topics such as personal values and goals, there are multiple indications that this technique may be well-suited to adolescents and children. Ambivalence is common in childhood and adolescence, so normalizing both the resistance and desire to change may reduce frustration in both the youth and the clinician (Mehlenbeck \& Wember, 2008). At the same time, adolescents are striving for autonomy, so encouragement of self-directed change is likely to be appealing (Baer \& Peterson, 2002). MI can be conducted in primary care offices or schools (Sindelar, Abrantes, Hart, Lewander, \& Spirito, 2004), increasing access for this population. MI has been conducted with the parent in cases where the child is too young to participate and the parent is responsible for much of the child's health behavior (e.g., pediatric obesity, dental care, passive smoke exposure for youth with asthma) (Resnicow, Davis, \& Rollnick, 2006). However, youth and adolescents often lack the independence and ability to make large lifestyle changes (Tevyaw \& Monti, 2004). Furthermore, without a long history of negative consequences for 
their risky behavior and perhaps without clear goals for the future, youth may not respond well to a discussion of how their current choices fit with their future aspirations (Tevyaw \& Monti, 2004).

\section{Moderators in the Child Literature}

Few moderators of the efficacy of MI have been analyzed in the child literature thus far, and knowledge of the situations and participants that would be most likely to benefit from MI is greatly needed (Erickson, Gerstle, \& Feldstein, 2005; Resnicow et al., 2006). Jensen and colleagues (2011) found that studies conducted on alcohol, tobacco, and drug interventions yielded relatively homogeneous effects (i.e., yielded a non-significant $Q$ statistic). They also demonstrated that effects were maintained over time, although effect sizes calculated less than six months after treatment were relatively larger than those calculated after six months posttreatment.

Suarez and Mullins (2008) proposed additional areas for moderator analysis, including a potential dose effect and an evaluation of whether MI is most effective as a stand-alone technique or as an adjunct to another intervention. Findings from the adult literature suggest there may be a dose effect, with greater intervention time yielding greater effect sizes; greater effect sizes when comparing MI groups to non-active controls than to other active treatments; larger effect sizes and more durable effects with some health domains; and larger effect sizes with minority populations, older participants, and with providers with greater displays of empathy (Burke et al., 2003; Dunn et al., 2001; Hettema \& Hendericks, 2010; Lundahl et al., 2010; Miller \& Baca, 1983; Rubak et al., 2005; Vasilaki et al., 2006). Together, these studies suggest that MI may be an effective intervention for substance-using adolescents and that its effectiveness appears to be consistent with the adult literature. 
Outside of substance use, MI has been applied to a range of child health domains, including diabetes, obesity and diet change, dental care, and reducing secondhand smoke in the homes of children with asthma (Suarez \& Mullins, 2008). In their systematic review of these studies, Suarez and Mullins (2008) reported that seven out of nine randomized control trials yielded positive findings of MI groups over comparison groups. Erickson (2005) reported empirical support for MI in dietary control, but a lack of sufficient evidence at this time to support it in other areas. Other researchers have likewise reported that the research in pediatric health-risk behavior areas is limited in quantity and quality, but that preliminary results are promising (Suarez, 2008; Wachtel \& Staniford, 2010). Erickson and colleagues (2005) said that MI is most promising in health care settings, but no systematic quantitative evaluation has been conducted to confirm this assertion.

A notable gap in the MI literature is a meta-analytic evaluation of this treatment as applied to health behavior change beyond substance abuse in youth. A literature review conducted by the author found no meta-analysis targeting this area. MI is being applied to youth populations because it has shown effectiveness in adult populations. Preliminary results in pediatric samples appear promising, but due to critical differences between adults and youth, such as lack of life experience, capacity for insight, and agency for change (i.e., ability to make changes in one's life), it is unreasonable to assume that effective treatments for adults would necessarily be effective for children. The present study is designed to (a) summarize the current literature evaluating the use of MI for changing health behaviors, (b) quantify the effectiveness of MI as compared to both active and passive controls, and (c) help explain the variation in the existing literature by including moderating variables in this analysis. By doing so, this study will 
provide direction for future research and assist practitioners in applying MI techniques most appropriately to their child clinical populations.

In light of previous meta-analyses, it is hypothesized that MI interventions for pediatric health behaviors will be associated with small to medium overall effect sizes. It is also hypothesized that MI will yield larger effect sizes when compared to control groups than active treatments, that greater time spent in treatment will be associated with larger effect sizes, and that effects will diminish over time but remain significant. Exploratory analyses will also determine whether MI performs differently alone versus in conjunction with another treatment, and whether different health populations yield different effects.

\section{Method}

\section{Literature Search}

Comprehensive literature searches using psychological, medical, and general databases (PsycINFO, PUBMED/MEDLINE, Social Work Abstracts, and Google Scholar) were used to identify interventions that reported using motivational interventions to change health behavior in youth. Searches were conducted using abbreviated and full keywords (motivation* enhancement OR interview*) AND (child OR adolescent OR youth). Further, backwards searching of reference lists of review articles and primary articles yielded additional relevant articles.

Dissertation Abstracts International was also searched to gather non-published data.

Additionally, the bibliography list available on the official MI website run by the Motivational Interviewing Network of Trainers (www.motivationalinterview.org) was searched for additional articles. Researchers who authored more than one included study were contacted individually to inquire if they had relevant unpublished data that they would be willing to share. After 
duplicates were excluded, this literature searched produced 353 articles that were screened for inclusion. This list is considered comprehensive as to fall of 2012.

\section{Inclusion/Exclusion Criteria}

All 353 article abstracts and articles were screened for potential inclusion in the study. Studies were retained at this point if they (a) mentioned MI or motivational enhancement therapy (MET), (b) involved an intervention using either comparison/treatment groups or pretest/posttest assessment, (c) used participants who were youth with an average age of $\leq 18$ years or parents of children in this age group, (d) reported target outcomes of pediatric health promotion, (e) were reported in English, and (f) reported enough information about results to calculate an effect size for the outcomes. Studies were excluded if mental health or substance abuse were the main health outcomes. Studies were included if MI was the sole treatment or if it was included in conjunction with another treatment. Articles were analyzed for potential inclusion by two trained research assistants and the author. Any disagreement was reconciled by discussion with the author until agreement was obtained.

\section{Coding of Studies}

Included articles were coded for study and participant characteristics by two independent research assistants as well as by the author. Any discrepancies identified were clarified by the author. Study characteristics that were coded included health outcome, groups compared (treatment vs. control), methodology, sample size, mean age, participant ethnicity, whether MI stood alone or was used with another treatment, number of sessions, duration of treatment, follow-up times, fidelity assessment, and study rigor. Study rigor was quantified based on study characteristics including reporting data on demographics, sample size, objectively measuring outcomes, assessing fidelity, etc., based on the rating scale used by Lundahl and colleagues 
(2010) (see Appendix for this rating scale). Effect sizes were calculated from differences in outcome variables between the intervention group and the control group or between pre- and post-intervention assessments. Raters achieved $96 \%$ reliability with each other.

\section{Statistical Approach}

Descriptive statistics of the studies included in the meta-analysis will first be presented, including range of health conditions, comparison groups, randomization procedures, follow-up length, fidelity to treatment, number of sessions, types of participants, and background of interventionist.

Effect sizes were calculated to measure the direction and magnitude of pediatric health behavior change as a result of MI interventions. These effect sizes were represented using Hedge's $g$. Hedge's $g$ was used rather than Cohen's $d$ because it yields more accurate results for studies with smaller sample sizes (Card, 2012). For studies that represented effect sizes using other metrics, data were converted to $g$ using standard procedures as recommended by Card (2012) based on the metric of the results of primary studies (e.g., from means and standard deviations, chi square contingency table, significance test, odds ratio, etc.). Studies reporting only a significant statistical association were assumed to be $p=0.05$ unless otherwise stated and will be assigned the minimum $g$ that would yield that level of significance. Likewise, if a study reports nonsignificance but did not report a specific $p$ value, the study was assigned a $g=0.00$. These methods represent a conservative measurement of overall effect size and thus may underrepresent the true effect size (Card, 2012).

Effect sizes were combined using a sample size weighted approach, where individual studies were weighted according to their amount of standard error, giving more weight to studies with larger sample sizes (Card, 2012). Multiple effect sizes from a single study were averaged to 
yield a single effect size per study to avoid violation of assumptions of independence. A weighted mean effect size was then calculated to combine weighted effect sizes from all studies. Cohen (1988) recommends categorizing effect sizes in the following manner: 0.20 as small, 0.50 as medium, and 0.80 as large. Confidence intervals were constructed for each study and intervals that did not contain zero were considered statistically significant and intervals that contained zero were considered not significant.

The Q statistic was calculated to determine the degree of heterogeneity in effect sizes across studies (Card, 2012). If heterogeneity was found, then moderators of amount of time in treatment, type of comparison group, health domain, study quality, and the presence of other additive treatment components were analyzed, as these are variables that have been relevant in the adult literature. A fixed-effects model was used because of our interest in evaluating a set of moderators. In this case, a fixed-effects approach is considered an acceptable approach, provided that moderators help to explain any residual heterogeneity and this heterogeneity is minimal (Card, 2012).

Evaluating categorical moderators in this meta-analysis used ANOVA techniques to partition between-study heterogeneity into heterogeneity between and within categories of interest (Card, 2012). A categorical moderator was considered significant if the amount of heterogeneity among studies (i.e., between-groups $Q$ statistic) became statistically nonsignificant after studies were partitioned into the categories of interest (e.g., health groups). However, this significance test is an omnibus test, indicating only a broad level of significance across the groups. Confidence intervals were constructed to indicate which categories are different from which other categories. It is suggested that around 20 studies be included in each category for optimal power to detect heterogeneity (Card, 2012). Because this meta-analysis only contained 
37 studies in total, lack of significant heterogeneity between categories may be due to a lack of power. For this reason, secondary analyses of multiple moderators were not conducted, as this would divide categories into even smaller groups.

Evaluating continuous moderators in this meta-analysis utilized weighted regression analyses, specifically regressing individual study effect sizes onto the continuous moderator in a weighted method so that studies with smaller standard errors contribute more to the regression equation (Card, 2012).

Studies were categorized across several dimensions for categorical moderator analyses. Some of these dimensions require description. Interventionist profession was determined using the descriptive data presented in the study. Studies were included in the "masters and doctoral level category" if these degrees were mentioned in the description of the interventionists. Studies were included in the "professional category" if no masters or doctoral degree was mentioned, but interventionists were described as health professionals (e.g., nurses, nutritionists, etc.). Studies were included in the community health worker category if no health profession or educational degree was described, but interventionists were described as being community members, health educators, health coaches, etc. If no descriptions were provided about the interventionists, the study was put in the "no data" category.

Regarding type of outcome, outcomes were considered physical if the measurement tool was directly measuring a marker of physical health (e.g., BMI, hemoglobin A1C, \# days breastfeeding, \# days with asthma symptoms, etc.). Outcomes were considered psychosocial if the tool was measuring a psychological factor related to health (e.g., motivation, self-efficacy, quality of life, self-concept, perceived barriers to change, etc.). 
Studies were considered to utilize a passive control group if the comparison group received no treatment (i.e., waitlist control); was the baseline measurement for the intervention group; or received some form of treatment as usual. Active control groups utilized an active form of treatment above and beyond treatment as usual (e.g., health education group, exercise group, problem-solving skills group, etc.).

As for health domain classification, several domains require description. The accident prevention category included any studies aimed at preventing injury or accident in children. The asthma category included studies that specifically stated they were designed to treat asthma symptoms and also studies designed to increase air quality in the homes of children at risk for respiratory problems. The dental health category included studies of targeting both children and parents brushing the children's teeth. The HIV/AIDS category included studies targeting specifically behaviors around treating and preventing contraction of this virus as well as studies that targeted STD prevention more widely. Lastly, the infant health category included studies promoting infant health directly (e.g., breastfeeding, maternal health), and also included studies aimed at preventing subsequent pregnancies in adolescent mothers for the health benefit of the first baby and the mother.

To assess for possible publication bias, both a failsafe $\mathrm{N}$ calculation and a funnel plot depiction was reported. A failsafe $\mathrm{N}$ calculation indicated the number of excluded studies averaging an effect size of zero that would have to exist in order for the overall effect size to be lowered to zero. A funnel plot is a scatterplot of effect sizes relative to sample sizes and a funnel shape of data points indicates a relative absence of publication bias. 


\section{Description of Studies}

\section{Results}

The literature search returned 37 studies that were eligible for inclusion based on stated criteria for this meta-analysis (Figure 1). Across these studies, 393 effect sizes were calculated. To prevent violations of independence, multiple effect sizes within the same study were averaged to create a single effect size per study. Not surprisingly, due to the large number of studies included in this analysis, the literature represents considerable diversity across study characteristics. Table 1 provides information about the characteristics of these studies.

Figure 1. PRISMA 2009 flow diagram for included studies.

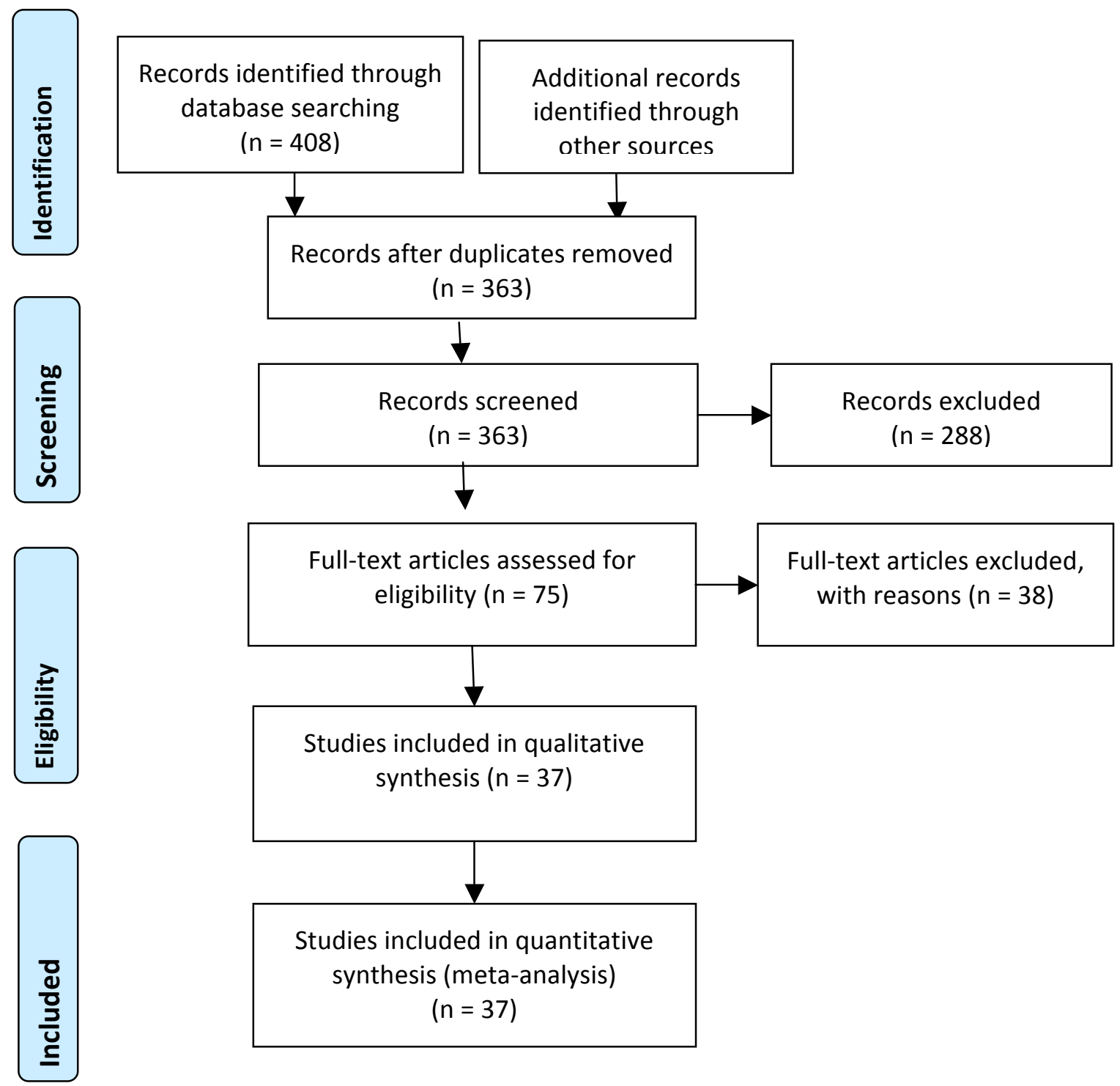


Intervention characteristics. The majority of the studies were published in the last five years, with the earliest study having been published in 2001, indicating that research in this field is rather new and growing rapidly.

Health conditions. Across the 37 identified studies, eight health conditions were targeted for intervention. Of the included studies, $32.4 \%(n=12)$ targeted pediatric obesity, $16.2 \%(n=6)$ targeted asthma, $13.5 \%(\mathrm{n}=5)$ targeted HIV/AIDS, $10.8 \%(\mathrm{n}=4)$ targeted Type I Diabetes, $8.1 \%(n=3)$ targeted infant health, $10.8 \%(n=4)$ targeted dental health, $2.7 \%(n=1)$ targeted accident prevention, $2.7 \%(\mathrm{n}=1)$ targeted sleep, and $2.7 \%(\mathrm{n}=1)$ targeted calcium intake.

Comparison groups. The majority of studies $(59 \% ; \mathrm{n}=22)$ compared MI to a passive control group (e.g., waitlist control, baseline measures). In contrast, $35.1 \%(n=13)$ of studies compared MI against another active treatment (e.g., moderate intensity version of the intervention, health education group, nutrition counseling group). Two studies (5.4\%) compared MI to both an active and a passive control group.

Studies differed in the quality of randomization procedures. True randomization procedures were used in 54\% of studies; matched groups or cluster randomization (e.g., by school, classroom, or medical practice) was used in $27 \%$ of studies; and comparison groups were not randomly assigned in 5\% of studies (but groups were tested for pretreatment equivalence across several variables). Fourteen percent of studies compared the intervention group's posttreatment scores to its pre-treatment scores.

About half of the studies $(48.6 \% ; \mathrm{n}=18)$ used MI as a stand-alone treatment and the other half $(51.4 \% ; \mathrm{n}=19)$ incorporated MI along with another form of treatment (e.g., exercise program, health education, parenting skills training, dietary intervention). 
Follow up. Average follow up length (measured as time since baseline) was 5.46 months (SD = 5.58), and follow-up ranged from no follow up to two years.

Treatment fidelity. Fidelity to treatment was discussed in $40.5 \%(\mathrm{n}=15)$ of studies. However, results of objective fidelity measurements (i.e., scores on the MITI 3.0) were reported in only $13.5 \%(\mathrm{n}=5)$ studies. When reported, fidelity to MI principles was generally high, with interventionists nearly always demonstrating proficiency in the domains of measurement (e.g., adherence to MI spirit, demonstration of empathy, open-ended questions, complex reflections).

Study rigor. Study rigor was measured using an 18-point methodological quality scale created by Lundahl and colleagues (2010). This scale was selected because it has been used in a similar meta-analysis and was the most comprehensive tool for this purpose identified. The components of this scale are described in the Appendix. Results indicated that the average total score on this scale was 10.78 , with a range of six to 15 . Results included the following: $70 \%$ of studies reported at least three demographic variables, $43 \%$ of studies had a follow up measurement after the post-intervention measurement, $24 \%$ of studies used blinded coders/data collectors, $70 \%$ of studies collected objective measurements, $16 \%$ of studies used a standardized intervention, and $89 \%$ of studies had over 20 participants per group. Seventy-three percent of studies presented enough information for an effect size to be calculated using means and standard deviations, which yields a more accurate effect size calculation than using a test statistic ( $27 \%$ of studies). In $62 \%$ of studies, outcomes came from at least two sources (e.g., objective measurement and self-report, or both parent and child reports), while for $27 \%$ of studies, self-report was the only source of data, and for $11 \%$ of studies, just a collateral source was used (e.g., objective report or parent report). 
Session characteristics. Both group and individual therapy sessions were utilized, with 56\% (n $=21)$ studies using group therapy MI sessions, $21.6 \%(\mathrm{n}=8)$ of studies using individual MI sessions, and 21.6\% $(\mathrm{n}=8)$ of studies incorporating both group and individual MI sessions. Average number of MI sessions was $4.53(\mathrm{SD}=4.21)$.

Participant characteristics. The average number of participants per study was 262.24 (SD = 540.042 ) with a range of 20 participants to over 4,000 participants. Although the targeted outcomes were pediatric focused, studies differed on which family members participated in the intervention. Results indicated that in $62.2 \%(\mathrm{n}=23)$ of studies the child participated in MI sessions alone, in $29.7 \%(\mathrm{n}=11)$ of studies the parent participated alone, and in $8.1 \%(\mathrm{n}=3)$ of studies the parent and child both participated in MI sessions. The child was on average 12.01 years of age $(\mathrm{SD}=6.04)$. Results also indicated that the average study's participants were $69.28 \%(\mathrm{SD}=6.04)$ non-Caucasian and $36.54 \%(\mathrm{SD}=24.86)$ male.

Interventionist characteristics. Interventionists had a wide range of backgrounds and education levels. Results demonstrated that $10.8 \%(n=4)$ studies used non-professional community members as interventionists, $27 \%(\mathrm{n}=10)$ studies used professional interventionists but specific information about educational training was not mentioned (e.g., nurses, dieticians), and 35.1\% $(\mathrm{n}=13)$ used Master's and doctoral level clinicians. Twenty-seven percent of studies $(\mathrm{n}=10)$ did not give information about the interventionist. 
Table 1. Selected study characteristics and average study effect sizes

\begin{tabular}{|c|c|c|c|c|c|c|c|c|c|c|}
\hline Author (Year) & $\begin{array}{l}\text { Total } \\
\text { Sample } \\
\text { Size (N) }\end{array}$ & $\begin{array}{l}\text { Study Effect } \\
\text { Size, } 95 \% \text { CI }\end{array}$ & Participant & $\begin{array}{l}\text { Average } \\
\text { Child } \\
\text { Age } \\
\text { (years) }\end{array}$ & $\begin{array}{l}\text { Health } \\
\text { concern }\end{array}$ & $\begin{array}{l}\text { Education level } \\
\text { of } \\
\text { interventionist }\end{array}$ & $\begin{array}{l}\text { Group or } \\
\text { Individual } \\
\text { Therapy }\end{array}$ & $\begin{array}{l}\text { \% of } \\
\text { Sample } \\
\text { Non- } \\
\text { Caucasian }\end{array}$ & $\begin{array}{l}\text { \# MI } \\
\text { sessions }\end{array}$ & $\begin{array}{l}\text { Follow- } \\
\text { up Period } \\
\text { (months) }\end{array}$ \\
\hline Ball (2011) & 20 & $\begin{array}{l}0.632 \\
(-0.266,1.530)\end{array}$ & Child & 15.6 & Obesity & HS & Both & 7 & 16 & 1 \\
\hline Barkin (2008) & 3284 & $\begin{array}{l}0.332 \\
(0.267,0.397)\end{array}$ & Parent & 6.5 & $\begin{array}{l}\text { Accident } \\
\text { prevention }\end{array}$ & MA & Group & 30 & 5 & 24 \\
\hline Barnet (2009) & 235 & $\begin{array}{l}0.357 \\
(0.034,0.679)\end{array}$ & Child & 17.0 & Infant health & HS & Group & 97 & 9 & 11 \\
\hline Black (2010) & 235 & $\begin{array}{l}0.067 \\
(-0.224,0.358)\end{array}$ & Child & 13.3 & Obesity & BA & Group & 97 & 12 & 1 \\
\hline Borrelli (2010) & 133 & $\begin{array}{l}0.074 \\
(-0.337,0.486)\end{array}$ & Parent & No data & Asthma & MA & Group & 97 & 2 & 1 \\
\hline Cain (2010) & 104 & $\begin{array}{l}0.119 \\
(-0.278,0.516)\end{array}$ & Child & 16.2 & Sleep & MA & Individual & No data & 4 & 1 \\
\hline Chan (2005) & 80 & $\begin{array}{l}0.177 \\
(-0.262,0.617)\end{array}$ & Parent & No data & $\begin{array}{l}\text { Smoking } \\
\text { cessation for } \\
\text { parents of sick } \\
\text { children } \\
\text { (asthma } \\
\text { group) }\end{array}$ & BA & Group & 100 & 1 & 6 \\
\hline Channon (2003) & 60 & $\begin{array}{l}2.300 \\
(1.345,3.254)\end{array}$ & Child & 15.0 & $\begin{array}{l}\text { Type } 1 \\
\text { Diabetes }\end{array}$ & BA & Group & No data & 4 & 2 \\
\hline Channon (2007) & 22 & $\begin{array}{l}0.353 \\
(-0.230,0.936)\end{array}$ & Both & 15.8 & $\begin{array}{l}\text { Type } 1 \\
\text { Diabetes }\end{array}$ & MA & Individual & 0 & 9 & 2 \\
\hline Davis (2011) & 38 & $\begin{array}{l}0.453 \\
(-0.342,1.247)\end{array}$ & Child & 16.0 & Obesity & No data & Both & 100 & 4 & 4 \\
\hline Flattum (2011) & 182 & $\begin{array}{l}1.738 \\
(0.987,2.489)\end{array}$ & Child & 15.7 & Obesity & No data & Individual & 75 & 7 & 0 \\
\hline $\begin{array}{l}\text { Freudenthal } \\
(2010)\end{array}$ & 72 & $\begin{array}{l}0.139 \\
(-0.303,0.571)\end{array}$ & Parent & No data & Dental health & No data & Group & No data & 1 & 9 \\
\hline $\begin{array}{l}\text { Halterman, } \\
\text { Riekert (2011) }\end{array}$ & 28 & $\begin{array}{l}0.570 \\
(0.083,1.137)\end{array}$ & Child & 13.6 & Asthma & BA & Group & 91 & 3 & 4 \\
\hline $\begin{array}{l}\text { Halterman, } \\
\text { Szilagyi (2011) }\end{array}$ & 530 & $\begin{array}{l}0.610 \\
(0.395,0.745)\end{array}$ & Both & 7.1 & Asthma & BA & Group & 100 & 1 & 24 \\
\hline Harrison 2007 & 240 & $\begin{array}{l}0.404 \\
(0.128,0.681)\end{array}$ & Parent & 1.0 & Dental health & HS & Group & 100 & 1 & 6 \\
\hline Ismail (2011) & 599 & $\begin{array}{l}0.076 \\
(-0.069,0.221)\end{array}$ & Parent & 4.0 & Dental health & MA & Both & 100 & 1 & 3 \\
\hline $\begin{array}{l}\text { MacDonell } \\
(2012)\end{array}$ & 44 & $\begin{array}{l}0.116 \\
(-0.475,0.708)\end{array}$ & Both & 15.1 & Obesity & No data & Group & 100 & 4 & 0.5 \\
\hline Manarino (2007) & 30 & $\begin{array}{l}0.597 \\
(0.079,1.114)\end{array}$ & Child & 12.5 & $\begin{array}{l}\text { Calcium } \\
\text { intake }\end{array}$ & MA & Individual & 33 & 2 & 6 \\
\hline $\begin{array}{l}\text { Naar-King, } \\
\text { Outlaw, }(2009)^{*}\end{array}$ & 87 & $\begin{array}{l}0.797 \\
(0.350,1.244)\end{array}$ & Child & 21.1 & HIV/AIDS & HS and MA & Group & 99 & 2 & 6 \\
\hline $\begin{array}{l}\text { Naar-King, } \\
\text { Parsons (2009)* }\end{array}$ & 186 & $\begin{array}{l}0.220 \\
(-0.100,0.539)\end{array}$ & Child & 20.5 & HIV/AIDS & MA & Group & 83 & 4 & 4 \\
\hline $\begin{array}{l}\text { Naar-King, } \\
(2006)^{*}\end{array}$ & 51 & $\begin{array}{l}0.576 \\
(-0.100,0.539)\end{array}$ & Child & 21.0 & HIV/AIDS & MA & Group & 97 & 4 & 9 \\
\hline $\begin{array}{l}\text { Neumark- } \\
\text { Sztainer (2010) }\end{array}$ & 356 & $\begin{array}{l}0.159 \\
(-0.054,0.372)\end{array}$ & Child & 15.8 & Obesity & No data & Both & 56 & 6 & 12 \\
\hline $\begin{array}{l}\text { Obarznack } \\
(2001)\end{array}$ & 663 & $\begin{array}{l}0.046 \\
(-0.118,0.209)\end{array}$ & Child & 9.0 & Obesity & MA & Both & 14 & 2 & 6 \\
\hline Olson (2008) & 284 & $\begin{array}{l}0.183 \\
(-0.100,0.466)\end{array}$ & Child & No data & Obesity & $\mathrm{BA}$ & Group & 4 & 1 & 6 \\
\hline $\begin{array}{l}\text { Resnicow, } \\
(2005)\end{array}$ & 123 & $\begin{array}{l}0.013 \\
(-0.356,0.383)\end{array}$ & Child & 13.6 & Obesity & $\mathrm{BA}$ & Individual & 100 & 6 & 2 \\
\hline Riekert (2011) & 37 & $\begin{array}{l}0.287 \\
(-0.171,0.745)\end{array}$ & Child & 12.5 & Asthma & $\mathrm{BA}$ & Group & 100 & 2 & 4.5 \\
\hline Robertson 2011 & 333 & $\begin{array}{l}0.469 \\
(0.216,0.723)\end{array}$ & Child & 15.0 & HIV/AIDS & No data & Both & 71 & 18 & 0 \\
\hline Schmiege (2009) & 484 & $\begin{array}{l}0.129 \\
(-0.092,0.351)\end{array}$ & Child & 15.8 & HIV/AIDS & No data & Individual & 64 & 1 & 6 \\
\hline Schwartz (2007) & 91 & $\begin{array}{l}0.275 \\
(-0.346,0.896)\end{array}$ & Parent & 5.3 & Obesity & MA & Both & No data & 1.5 & 1 \\
\hline Seid (2011) & 26 & $\begin{array}{l}0.781 \\
(-0.019,1.581)\end{array}$ & Child & 15.0 & Asthma & MA & Group & 89 & 2 & 6 \\
\hline
\end{tabular}




\begin{tabular}{|c|c|c|c|c|c|c|c|c|c|c|}
\hline $\begin{array}{l}\text { Taveras, } \\
\text { Gortmaker } \\
\text { (2011) }\end{array}$ & 84 & $\begin{array}{l}0.192 \\
(0.004,0.380)\end{array}$ & Parent & 0.25 & Obesity & MA & Group & 43 & 4 & 12 \\
\hline $\begin{array}{l}\text { Taveras, } \\
\text { Blackburn (2011) }\end{array}$ & 475 & $\begin{array}{l}-0.002 \\
(-0.189,0.186)\end{array}$ & Parent & 4.5 & Infant health & MA & Both & 26 & 4 & 6 \\
\hline Viner (2003) & 77 & $\begin{array}{l}2.745 \\
(2.082,3.408)\end{array}$ & Child & 13.3 & $\begin{array}{c}\text { Type I } \\
\text { Diabetes }\end{array}$ & No data & Individual & No data & 6 & 6 \\
\hline Wang (2010) & 44 & $\begin{array}{l}-0.551 \\
(-1.153,0.051)\end{array}$ & Child & 15.0 & $\begin{array}{l}\text { Type } 1 \\
\text { Diabetes }\end{array}$ & No data & Group & 32 & 2 & 0 \\
\hline Wilhelm 2006 & 73 & $\begin{array}{l}0.222(-0.239 \\
0.682)\end{array}$ & Parent & 0.25 & $\begin{array}{l}\text { Infant } \\
\text { health }\end{array}$ & BA & Group & 12 & 3 & 3 \\
\hline Wilson (2002) & 53 & $\begin{array}{l}0.400(-0.242 \\
1.041)\end{array}$ & Child & 13.0 & Obesity & No data & Individual & 100 & 12 & 1 \\
\hline
\end{tabular}

* These studies were included even though the mean age was above 18 because a substantial percentage of the age range was within adolescence 
Overall effect size. The overall effect size for MI interventions targeting pediatric health behavior change was statistically significant $(g=0.282,95 \% \mathrm{CI}[0.242-0.323], \mathrm{SE}=0.021, \mathrm{n}=$ 37). This finding is slightly larger than Cohen's classification of a "small effect size" (Cohen, 1988). In fact, only $2.7 \%$ of studies yielded a negative effect size and the bottom $25 \%$ of studies ranged from a negative effect size to an effect size of just over 0.1 , indicating that even the most ineffective studies were still largely more effective than the alternative. Almost all studies in this meta-analysis (95\%) yielded improved outcomes as compared to the alternate group or to the baseline. The top $25 \%$ of studies yielded effect sizes of 0.57 and above, classified by Cohen as "medium" to "large," indicating a sizable improvement over the comparison group. Individual studies contributed to the weighted overall effect size as depicted in the forest plot in Figure 2. Forest plots depict each study's effect size, length of its confidence interval, and contribution to the overall effect size. Study weight is largely determined by sample size, with studies with more participants having more weight. This figure depicts the dispersion of individual studies around the overall effect size $(g=0.282)$.

Given the range and diversity of study variables, this overall effect size likely is too broad to capture the many factors influencing study effect sizes. Not surprisingly, these studies yielded a significant $Q$-statistic, indicating that the studies included in the meta-analysis displayed a more heterogeneous group of effect sizes than would be expected on the basis of chance alone $(Q$ $=159.0588, \mathrm{p} \leq .001)$. This finding indicates that moderating variables, such as participant, session, and practitioner characteristics, are significantly influencing study effect sizes, and are discussed below. 
Chan (2005), Asthma

Halterman, Szilagyi (2011), Asthma

Halterman, Riekert (2011), Asthma

Figure 2. Fore

Riekert (2011), Asthma Seid (2011), Asthma

Manarino (2007), Calcium Intake

Freudenthal (2010), Dental Health

Harrison (2007), Dental Health

Ismail (2011), Dental Health

Weinstein (2006), Dental Health

Barkin (2008), Accident Prevention

Naar-King, Outlaw (2009), AIDS

Naar-King, Parsons (2009), AIDS

Naar-King (2006), AIDS

Robertson (2011), AIDS

Schmiege (2009), AIDS

Barnet (2009), Infant Health

Taveras, Blackburn (2011), Infant Health

Wilhelm (2006), Infant Health

Ball (2011), Obesity

Black (2010), Obesity

Flattum (2011), Obesity

MacDonell (2012), Obesity

Neumark-Sztainer (2010), Obesity

Obarznack (2001), Obesity

Olson (2008), Obesity

Resnicow (2005), Obesity

Schwartz (2007), Obesity

Taveras, Gortmaker (2011), Obesity

Wilson (2002), Obesity

Davis (2011), Obesity

Cain (2010), Sleep

Moderator analyses

Moderator var

sessions, length of fol

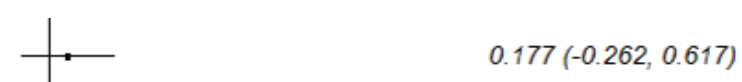

$0.570(0.395,0.745)$

$0.610(0.083,1.137)$

$0.287(-0.171,0.745)$

$0.781(-0.019,1.581)$

$0.597(0.079,1.114)$

$0.139(-0.303,0.581)$

$0.404(0.128,0.681)$

$0.076(-0.069,0.221)$

$0.562(0.282,0.842)$

$0.332(0.267,0.397)$

$0.797(0.350,1.244)$

$0.220(-0.100,0.539)$

$0.576(0.016,1.137)$

$0.469(0.216,0.723)$

$0.129(-0.092,0.351)$

$0.357(0.034,0.679)$

$-0.002(-0.189,0.186)$

$0.222(-0.239,0.682)$

$0.632(-0.266,1.530)$

$0.067(-0.224,0.358)$

$1.738(0.987,2.489)$

$0.116(-0.475,0.708)$

$0.159(-0.054,0.372)$

$0.046(-0.118,0.209)$

$0.183(-0.100,0.466)$

$0.013(-0.356,0.383)$

$0.275(-0.346,0.896)$

$0.192(0.004,0.380)$

$0.400(-0.242,1.041)$

$0.453(-0.342,1.247)$

$0.119(-0.278,0.516)$

$2.300(1.345,3.254)$

$0.353(-0.230,0.936)$

$2.745(2.082,3.408)$

$-0.551(-1.154,0.051)$

characteristics (e.g., age, participant). These variables were chosen based on findings from

previous MI meta-analyses (e.g., Dunn et al., 2001; Burke et al., 2003; Hettema \& Hendericks,

2010; Lundahl et al., 2010; Rubak et al., 2005; Vasilaki et al., 2006). A statistically significant $Q$ 
statistic indicated that individual study effect sizes were heterogeneous, and thus that testing for moderation was warranted. Both continuous and categorical moderators were assessed and the results are described separately due to differing statistical procedures.

Continuous moderators. Continuous moderators analyzed in this meta-analysis were average child age, percentage male, percentage non-Caucasian, number of follow-up sessions, length of follow-up, and study quality. Table 2 demonstrates results from these analyses. None of these continuous moderators yielded a sizable or statistically significant $R^{2}$ value. For number of sessions, $70 \%$ of studies utilized four or less sessions of MI, seemingly without a loss of impact.

Table 2. Summary table of continuous moderator analyses

\begin{tabular}{llll} 
Continuous Moderators & $\mathbf{R}^{2}$ value & $\boldsymbol{p}$ value & Beta \\
\hline Age & 0.023 & 0.396 & 0.153 \\
Percent Male & 0.002 & 0.816 & -0.041 \\
\% Non-Caucasian & 0.034 & 0.313 & 0.184 \\
\# MI sessions & 0.016 & 0.460 & 0.125 \\
Follow up length & 0.012 & 0.513 & 0.111 \\
Study quality & 0.025 & 0.355 & -0.021 \\
\hline
\end{tabular}

Categorical moderators. Categorical moderators tested in this meta-analysis included provider education level, participant (e.g., child, parent, both), group/individual therapy, MI as standalone/component, type of control group, and fidelity to treatment. Results are depicted in Table 3. Practitioner profession, participant, and fidelity to treatment were statistically significant moderators, because they reduced the amount of between-groups variance to non-significant levels (see column 5, Table 3). The other categories were non-significant at the omnibus level, as they left variance unexplained, but groups within the category may still have accounted for 
significant variance. Results of categorical moderator analyses are presented numerically in Table 3 and graphically in Figure 3.

Practitioner Profession Characteristics. Community health workers demonstrated the largest effect size $(g=0.491 ; 95 \%$ CI [0.334-0.649]), followed by professional interventionists (e.g., nurses, nutritionists) $(g=0.361 ; 95 \% \mathrm{CI}[0.252-0.470])$, and interventionists with Master's and Doctorate degrees demonstrated the lowest effect size $(g=0.240,95 \%$ CI [0.191-0.289]). These results suggest that community health workers are statistically significantly more successful at eliciting client behavior change than interventionists with Master's and doctorate degrees, but not statistically distinct from professional interventionists.

Participant Characteristics. Studies that were partitioned based on whether they conducted MI with the child, parent, or with both child and parent. Studies which conducted MI with both the child and parent yielded the largest effect sizes ( $g=0.586,95 \%$ CI [0.370-0.752]), demonstrating that conducting MI with both parent and child is more effective than conducting MI with either parent or child individually.

Session characteristics. Both individual and group MI sessions appeared to be similarly effective; however, there was more variability among studies using individual sessions than in studies using group session studies (individual $g=0.361,95 \% \mathrm{CI}[0.074-0.506]$, group $g=$ $0.336,95 \%$ CI $[0.287-0.385])$.

Intervention Characteristics. Studies of MI as a stand-alone treatment were not significantly different than studies that used MI with other treatment components, although MI alone tended to demonstrate greater effect sizes (alone $g=0.324,95 \% \mathrm{CI}$ [0.271-0.377]; component $g=$ $0.224,95 \%$ CI [0.162-0.287]). Psychosocial outcomes of studies tended to demonstrate larger 
effect sizes than physical outcomes of studies, but the results were not statistically significant (psychosocial $g=0.221,95 \%$ CI [0.248-0.194], physical $g=0.184,95 \%$ CI $[0.168-0.200]$ ).

Studies that mentioned assessing fidelity to treatment were not distinct from studies that did not assess fidelity (checked $g=0.217,95 \%$ CI [0.126-0.308], not checked $g=0.298,95 \%$ CI [0.2530.343]). Finally, as expected, effect sizes tended to be greater when MI was compared to a passive control than to an active control (passive control $g=0.301,95 \%$ CI [0.257-0.346]), active control $g=0.189,95 \% \mathrm{CI}[0.099-0.279])$.

Table 3. Summary table of categorical moderator analyses

\begin{tabular}{|c|c|c|c|c|}
\hline Categorical Moderator & Effect Size (95\% CI) & $\begin{array}{l}Q \text { test of } \\
\text { heterogeneity }\end{array}$ & $\begin{array}{l}\text { \# Studies } \\
\text { in group }\end{array}$ & $\begin{array}{l}\text { Between groups } \\
Q \text { statistic }\end{array}$ \\
\hline Interventionist Profession & & & & 4.516 \\
\hline $\begin{array}{l}\text { - Community Health } \\
\text { Worker }\end{array}$ & $0.491(0.334,0.649)$ & 3.094 & 4 & \\
\hline - Professional & $0.361(0.252,0.470)$ & $32.496 * * *$ & 10 & \\
\hline - $\quad$ Masters + & $0.240(0.191,0.289)$ & $30.825 * *$ & 13 & \\
\hline - No data & $0.312(0.198,0.426)$ & $80.748 * * *$ & 10 & \\
\hline Participant & & & & 13.825 \\
\hline - Child & $0.257(0.185,0.370)$ & $104.742 * * *$ & 23 & \\
\hline - $\quad$ Parent & $0.266(0.215,0.317)$ & $25.605^{*}$ & 11 & \\
\hline - Both & $0.586(0.421,0.752)$ & $14.829 * * *$ & 3 & \\
\hline Group vs. Individual Therapy & & & & $22.037 * * *$ \\
\hline - Group & $0.336(0.287,0.385)$ & $52.047 * * *$ & 21 & \\
\hline - Individual & $0.361(0.074,0.506)$ & $72.431 * * *$ & 8 & \\
\hline - Both & $0.117(0.037,0.197)$ & 12.364 & 8 & \\
\hline Alone vs. Component & & & & $5.606^{*}$ \\
\hline
\end{tabular}


- Alone

- Component

Outcome Type $\dagger$

- Physical

- Psychosocial

Type of Control Group

- Active Control

- Passive Control

Fidelity to Treatment

- Checked

- Not Checked

Health Domain

- Accident prevention

- Asthma

- Calcium intake

- Dental health

- HIV/AIDS

- Infant health

- Obesity

- Sleep

- Type 1 Diabetes

$\begin{array}{llll}0.324(0.271,0.377) & 39.284^{* *} & 18 & \\ 0.224(0.162,0.287) & 114.039^{* * *} & 19 & 5.418^{*} \\ & & & \\ 0.184(0.168,0.200) & 4205.273^{* * *} & 261 & 57.486^{* * *} \\ 0.221(0.194,0.248) & 1994.482^{* * *} & 130 & \\ & & & 2.403 \\ 0.189(0.099,0.279) & 23.013^{*} & 12 & \\ 0.301(0.257,0.346) & 78.431^{* * *} & 25 & 46.165^{* * *} \\ & & & \\ 0.217(0.126,0.308) & 39.502^{* * *} & 15 & \\ 0.298(0.253,0.343) & 117.096^{* * *} & 22 & \\ & & & \\ 0.293(0.267,0.397) & \text { N/A } & 1 & \\ 0.444(0.454,0.435) & 10.544 & 7 & \\ 0.597(0.080,1.114) & \text { N/A } & 1 & \\ 0.213(0.207,0.220) & 11.366^{* *} & 4 & \\ 0.332(0.323,0.342) & 9.718^{*} & 5 & \\ 0.153(0.136,0.171) & 2.979 & 3 & \\ 0.150(0.146,0.154) & 21.656^{*} & 12 & \\ 0.119(-0.278,0.516) & \text { N/A } & 1 & \\ 0.914(0.858,0.970) & 63.668^{* * *} & 4 & \end{array}$

$\dagger$ Because an individual study often reported on multiple outcomes, all effect sizes were included in this analysis. 
Figure 3. Forest plot of categorical moderators

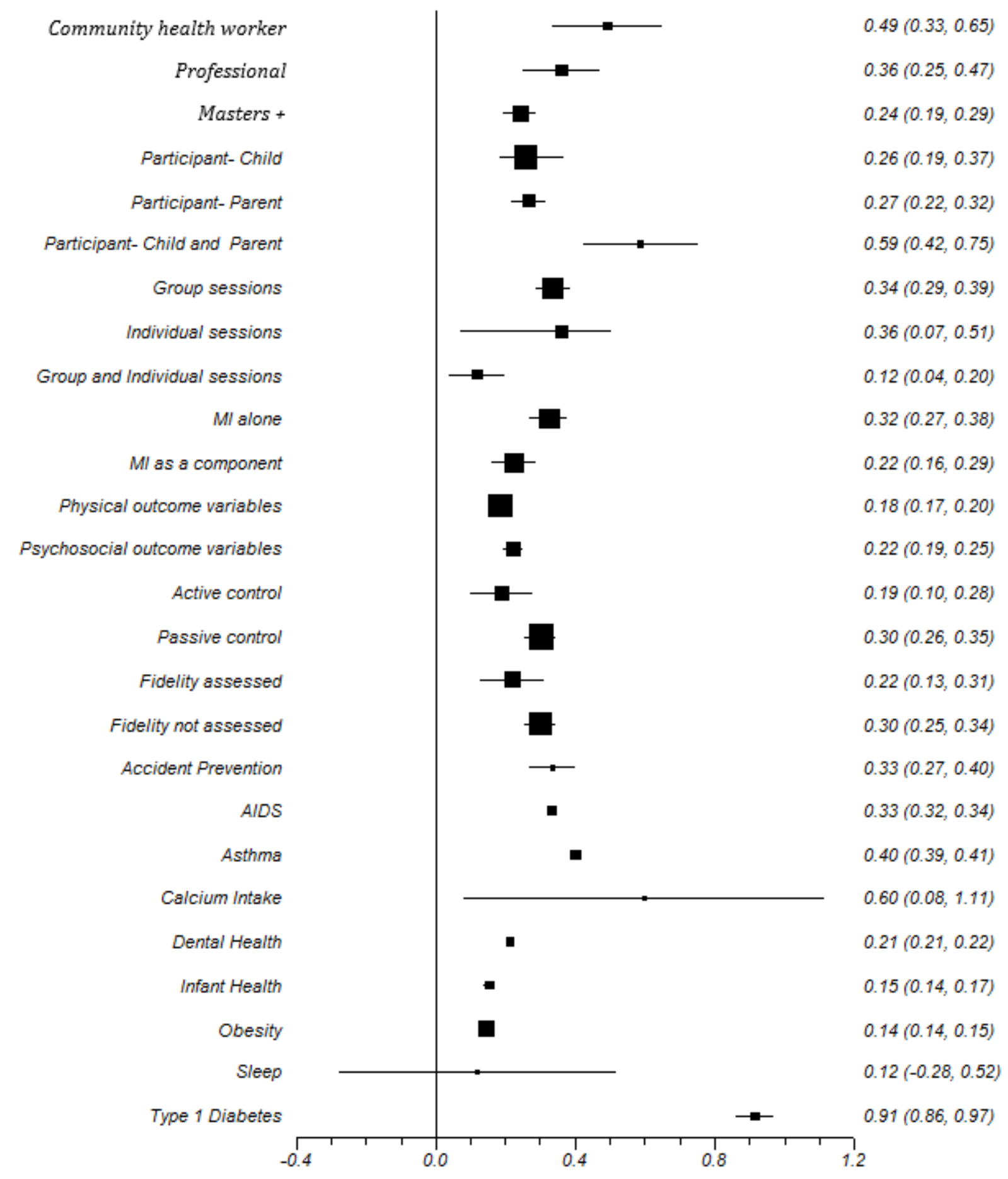


Fail-Safe $N$ Calculation. Because studies with null findings are more likely to remain unpublished than studies with significant findings, there is a concern that the overall effect size of a meta-analysis may be an overly positive estimation of the true effect size in the population. In this study, a fail-save $N$ calculation demonstrated that approximately 1,555 studies with an effect size of zero would be required to bring the observed overall effect size to zero (Rosenthal, 1979). Further evidence against publication bias is depicted in the funnel plot in Figure 4. A more-or-less funnel shaped plot provides support that studies were not selectively published based on significance.

Figure 4. Funnel plot of study sample size and effect size

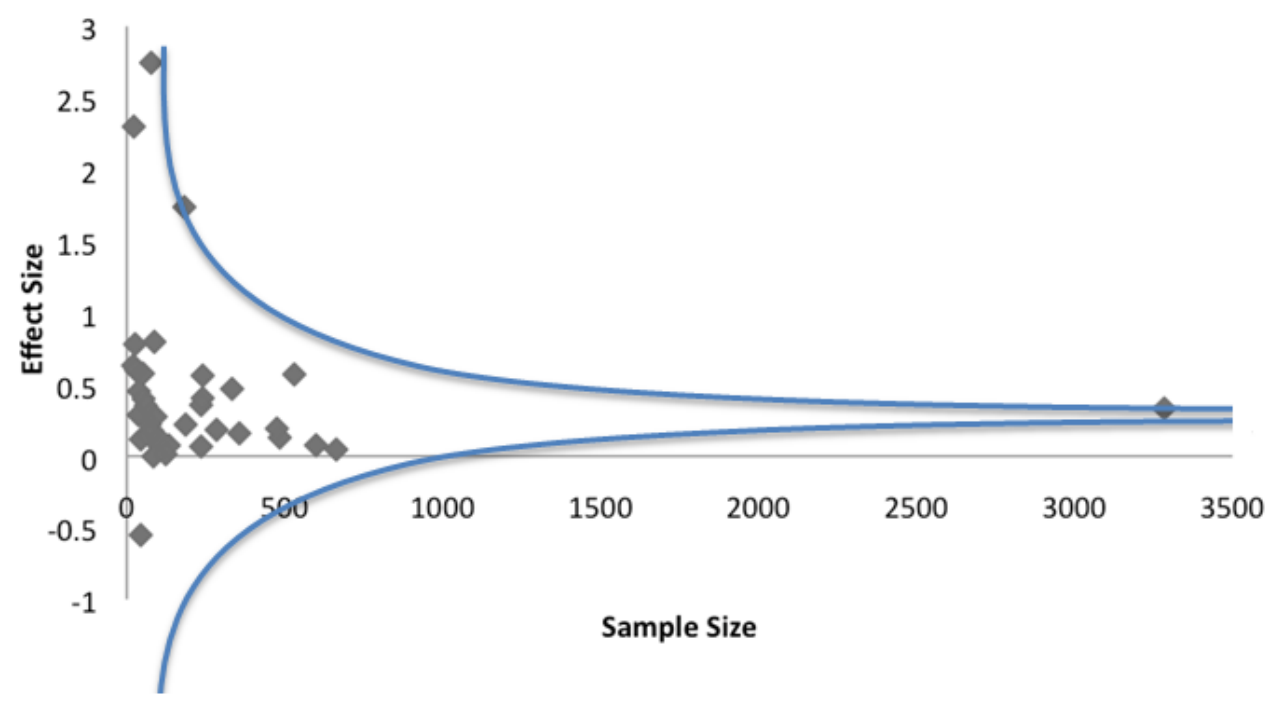




\section{Discussion}

Motivational interviewing is a clinical technique intended to help clients make behavioral changes in their lives. Originally designed for adult substance users, it has since been adapted for use across a range of health behaviors and a diversity of clinical populations. This metaanalysis is the first to examine the effectiveness of MI for health behavior change in pediatric populations. Pediatric health domains currently utilizing MI varied widely and included asthma, HIV/AIDS, dental health, Type 1 Diabetes, infant health, obesity, calcium intake, accident prevention, and sleep. Results from this meta-analysis suggest that MI produces a small but significant effect size across a range of child health behaviors $(g=0.282)$, indicating that overall, MI out-performed the alternative. Furthermore, effects of MI appear to be durable, as there was not evidence of a statistically significant decrease in effect size over follow up time points, consistent with previous findings (e.g., Jensen et al., 2001; Lundahl et al., 2010, Heckman et al., 2010). Thus as a whole, the results suggest that MI is an effective and appropriate technique to use for pediatric health behavior change.

These findings are consistent with previous meta-analyses demonstrating the effectiveness of MI to target substance use behavior change (e.g., Jensen et al., 2011; Burke et al., 2004; Rubak et al., 2005), although the estimated effect size was slightly greater in the present study. Considering the inherent physiological, social, and behavioral challenges of changing addictive behaviors, it may not be surprising that behavior change for other types of health behavior change may be more successful than substance use behavior change. It may be that pediatric health behaviors have more supportive systems in place that work to sustain change (e.g., families, schools, medical providers) (Ellis, Naar-King, Chen, Moltz, Cunningham, \& Idalski-Carcone, 2012). Also, stigma around adolescent substance use has been found to be a 
significant barrier to treatment (Wisdom, Cavaleri, Gogel, \& Nacht, 2011); perhaps there tends to be less stigma around health behaviors in youth, making it easier to seek support from others. When comparing effectiveness across health domains, it is most notable that all health domains that included more than one study yielded statistically significant positive effect sizes, largely consistent with previous research (e.g., Lundahl et al., 2010). MI was most effective in asthma, calcium intake, and Type 1 Diabetes domains, yielding medium-large effect sizes. Interventions targeting Type 1 Diabetes behaviors yielded the largest overall effect size ( $g=$ 0.914). However, of the four studies in this domain, two studies yielded negative or nearly null effect sizes and two studies yielded very large effect sizes (i.e., $g \geq 2.0$ ). This substantial discrepancy warrants caution and further investigation before MI is applied broadly to this population. Overall, MI appears to be applicable to a range of child health concerns and should continue to be applied and assessed with new populations.

Notably, MI seems to be effective for both physical and psychosocial health outcomes. This finding is encouraging because it demonstrates that not only does this technique increase the psychosocial health variables MI targets directly (e.g., motivation for change, self-efficacy for health behaviors), MI also increases the health behaviors themselves to the same extent, which is often the ultimate goal of an intervention. This finding is consistent with the self-efficacy component of MI and client-centered approaches in general that the individual already has within themselves the ability to make positive changes (Miller \& Rollnick, 2002).

Although consistent with previous meta-analyses (e.g., Jensen et al., 2001, Lundahl et al., 2010), it is still noteworthy to discuss our finding that a majority of practitioners did not have a graduate-level degree, and that non-professional practitioners may demonstrate greater effectiveness as compared to their professional colleagues. These results suggest that 
community health workers may be most successful at using MI techniques to encourage behavioral change with families, likely due to their unique understanding of cultural and community-specific concerns (Nemcek \& Sabatier, 2003). While this meta-analysis did not include community health workers in Latino communities, our findings are reminiscent of the concept of "Promotores de Salud"- community members who participate in health promotion initiatives (U.S. Department of Health and Human Services, 2007). In several community based studies, "Promotores de Salud" have been shown to be particularly successful at increasing health awareness and promoting treatment in populations that would otherwise have limited access to health care (Stacciarini, Rosa, Ortiz, Munari, Uicab, \& Balam, 2012), likely because they share the community, ethnicity, language, socioeconomic status, and life experiences of participants (U.S. Department of Health and Human Services, 2007). These findings are consistent with the Miller and Rollnick's assertion that the therapist's display of empathy and the quality of the therapeutic alliance are the most essential components of behavior change (Miller \& Rollnick, 2002).

Similarly, matching cultural and ethnic factors between the interventionist and the client is likely important and may contribute to the increased success of community health workers as compared to their professional colleagues. Since the average study had $70 \%$ of non-Caucasians participating and because the Master's and doctoral level interventionists were likely Caucasian, it is probable that a cultural mismatch occurred, which potentially reduced the effectiveness of the therapeutic intervention. Future research should examine more specifically the role of profession or training and the role of ethnic and cultural background match in MI effectiveness.

Examination of several moderators of therapeutic effects provide guidance for the adaptation of MI specifically to child and adolescent patient populations. A clear finding is that 
MI is most effective when conducted with both parent and child, and loses some effectiveness when conducted with either parent or child alone. This finding is consistent with the conceptual shift in pediatric psychology towards family-based lifestyle interventions that promote sustainable health behaviors for the whole family (Kitzmann et al., 2010). Obviously, in some circumstances (e.g., infant health concerns), MI with a single participant is appropriate and adequate.

A surprising finding was that MI appeared to be most effective as a stand-alone treatment rather than as an adjunct to another form of treatment. This result is noteworthy considering that about half of the studies in this meta-analysis included MI as a component of broader treatment. It is possible that the nature of the condition explains this finding; outcomes that are harder to achieve (i.e., BMI reduction, hemoglobin A1C reduction) may have been more likely to be targeted in multiple component interventions. It could also be that if not handled thoughtfully, the more prescriptive interventions that accompanied client-centered MI (e.g., health education) actually reduced the effectiveness of MI by presenting potentially conflicting theoretical orientations.

Numerous moderators were non-significant with this population (e.g., gender, age of participant, ethnicity, study quality, number of sessions). Of these variables, gender has been consistently found to be unrelated to effect size (e.g., Hettema \& Hendricks, 2010; Vasilaki et al., 2006), suggesting that future studies should not be concerned with this factor. However, other continuous moderators (e.g., age of participant, ethnicity, study quality, number of sessions) were not consistent with the previous MI literature and are worthy of continued examination. As discussed above, health behaviors may be easier and less complicated to 
change than addictive behaviors. It is also likely that these non-significant moderators represent different processes at work in children than among adults.

For instance, contrary to previous findings with adult populations (i.e., Lundahl et al., 2010; Rubak et al., 2005), we did not find evidence of a statistically significant dose effect, indicating that MI did not become more successful with more sessions. In fact, most studies used four or less sessions of MI, and brief treatments seemed to be just as effective as longer treatments. This finding is consistent with MI's original conceptualization as a brief intervention, but suggests that MI may function differently here than with adults' substance use behaviors. Perhaps health behaviors change fairly quickly and smoothly in just a few sessions. Also, due to advantages of youthfulness (e.g., a shorter history of engaging in the problematic behavior), child behaviors may be less ingrained and more readily changed as compared to adult behaviors that may have decades of habituation.

Thus it is possible that these non-significant moderators represent different processes at work with children, perhaps due to younger age, the family context, shorter behavioral history, and potential range of support systems (e.g., school, sports, peers, etc.). Also possible is that these null findings are due to a lack of power in this analysis to detect small effects due to our sample size. As more studies are conducted with pediatric populations, it will become more clear which moderators are relevant and the mechanisms of how they impact effectiveness of MI.

Finally, an area of concern for the field is the lack of attention to study quality and measurement of fidelity to treatment. The average study earned only slightly above half of the possible points for study quality and more than half of the studies did not measure fidelity to treatment. Without serious attention paid to conducting highly rigorous studies that are adherent to the principles of MI, our conclusions remain limited. Similarly, future studies should consider 
measuring process variables to clarify mechanisms of change in MI interventions. A better understanding of the MI change process would give guidance as to the key elements of MI interventions and lead to more consistently positive outcomes.

This meta-analysis has several limitations. While this analysis is the largest so far to examine MI in children, it did not contain enough studies to examine secondary moderators (i.e. interacting factors) and was likely underpowered to detect small moderating effects. Also, due to the emerging nature of this field, this meta-analysis included studies of a range of quality (e.g., used pre-post design, had small samples, etc.). Furthermore, due to the broad scope of this analysis, effect sizes were calculated for a wide range of physical and psychosocial health outcomes. While we believe this approach was appropriate at this stage of this field's development, it limits our understanding of MI's effectiveness for any specific outcome. As more studies are conducted in this field, more detailed analyses can be undertaken.

In summary, the findings of this meta-analysis suggest that MI is an appropriate and effective technique for pediatric populations across a range of health domains and appears to be particularly effective within the family context. MI participants show more substantial behavior changes as compared to both other active treatments and waitlist controls. That MI is at least as effective as other active treatments is exciting, especially considering that MI may offer several benefits as compared to traditional treatments. Our findings suggest that in pediatric populations, MI exerts its effects in a relatively brief period of time and can be delivered effectively by well-trained community health workers, reducing costs to the agency and client. Cost effectiveness measurements could be included in future studies. However, less than half of the studies in this analysis evaluated MI against strong comparison groups. Future studies should continue to rigorously compare MI to other established treatments. 


\section{References}

Amrhein, P.C. (1992). The comprehension of quasi-performance verbs in verbal commitments: New evidence for commitment language. Journal of Memory and Language, 31, 756784.

Burke, B. L., Arkowitz, H., \& Menchola, M. (2003). The efficacy of motivational interviewing: A meta-analysis of controlled clinical trials. Journal of Consulting and Clinical Psychology, 71, 5, 843-861.

Burke, B. L., Dunn, C. W., \& Atkins, D. C., (2004). The emerging evidence base for motivational interviewing: A meta-analytic and qualitative inquiry. Journal of Cognitive Psychotherapy, 18, 309-322.

Butler, C. C., Rollnick, S., Cohen, D., Russel, I., Bachmann, M., \& Stott, N. (1999). Motivational consulting versus brief advice for smokers in general practice: A randomized trial. British Journal of General Practice, 49, 611-616.

Baer, J. S., \& Peterson, P. L. (2002). Motivational interviewing with adolescents and young adults. In W. R. Miller \& S. Rollnick (Eds), Motivational interviewing: Preparing people for change ( $2^{\text {nd }}$ ed.) (320-332). New York: Guilford Press.

*Ball, G. D. C., Mackenzie-Rife, K. A., Newton, M. S., Alloway, C. A., Slack, J. M., Plotnikoff, R. C., \& Goran, M. I. (2011). One-on-one lifestyle coaching for managing adolescent obesity: Findings from a pilot, randomized controlled trial in a real-world, clinical setting. Paediatric Child Health, 16(6), 345-350.

Battjes, R. J., Gordon, M. S., O’Grady, K. E., \& Kinlock, T. W. (2004). Predicting retention of adolescents in substance abuse treatment. Addictive Behaviors, 29, 1021-2027. 
*Barkin, S. L., Finch, S. A., Ip, E. H., Scheindlin, B., Craig, J. A., Steffes, J., . . Wasserman, R. C. (2008). Is office-based counseling about media use, timeouts, and firearm storage effective? Results from a cluster-randomized, controlled trial. Pediatrics, 122(15), e15e25.

*Barnet, B., Liu, J., DeVoe, M., Duggan, A. K., Gold, M. A., \& Pecukonis, E. (2009). Motivational intervention to reduce rapid subsequent births to adolescent mothers: A community-based randomized trial. Annals of Family Medicine, 7(5), 436-445.

*Black, M. M., Hager, E. R., Le, K., Anliker, J., Arteaga, S. S., DiClemente, C., . . Wang, Y. (2010). Challenge! Health promotion/obesity prevention mentorship model among urban, black adolescents. Pediatrics, 126, 280-288.

*Borrelli, B., McQuaid, E. L., Novak, S. P., \& Hammond, S. K. (2010). Motivating Latino caregivers of children with asthma to quit smoking: A randomized trial. Journal of Consulting and Clinical Psychology, 78(1), 34-43.

*Cain, N., Gradisar, M., \& Moseley, L. (2011). A motivational school-based intervention for adolescent sleep problems. Sleep Medicine, 12, 246-251.

Card, N. A. (2012). Applied meta-analysis for social science research. New York: The Guilford Press.

Carey, K. B., Scott-Sheldon, L. A. J., Carey, M. P., \& DeMartini, K. S. (2007). Individual-level interventions to reduce college student drinking: A meta-analytic review. Addictive Behaviors, 32, 2469-2494.

*Chan, S. S. C., Lam, T. H., Salili, F., Leung, G. M., Wong, D. C. N., Botelho, R. J... Lau, Y. L. (2005). A randomized controlled trial of an individualized motivational intervention 
on smoking cessation for parents of sick children: A pilot study. Applied Nursing Research, 18, 178-181.

${ }^{*}$ Channon, S., Smith, V. J., \& Gregory, J. W. (2003). A pilot study of motivational interviewing in adolescents with diabetes. Archives of Disease in Childhood, 88, 680-683.

*Channon, S. J., Huws-Thomas, M. V., Rollnick, S., Hood, K., Cannings-John, R. L., Rogers, C., \& Gregory, J. W. (2007). A multicenter randomized controlled trial of motivational interviewing in teenagers with diabetes. Diabetes Care, 30(6), 1390-1395.

Cohen, J. (1988). Statistical power analysis for the behavioral sciences $\left(2^{\text {nd }}\right.$ ed.). Hillsdale, NJ: Erlbaum.

*Davis, J. N., Gyllenhammer, L. E., Vanni, A. A., Meija, M., Tung, A., Schroeder, E. T., ... Goran, M. I. (2011). Startup circuit training program reduces metabolic risk in Latino adolescents. Medicine and Science in Sports and Exercise, 43(11), 2195-2203.

DiRosa, L. C. (2009). Motivational interviewing to treat overweight/obesity: A meta-analysis of relevant research. Dissertation Abstracts International Section A: Humanities and Social Sciences, 71 .

Duff, A. J. A., \& Latchford, G. J. (2010). Motivational interviewing for adherence problems in cystic fibrosis. Pediatric Pulmonology, 45, 211-220.

Dunn, C., DeRoo, L., \& Rivara, F. P. (2001). The use of brief interventions adapted from MI across behavioral domains: A systematic review. Addiction, 96, 1725-1743.

Ellis, D. A., Naar-King, S., Chen, X., Moltz, K., Cunningham, P. B., \& Idalski-Carcone, A. (2012). Multisystemic therapy compared to telephone support for youth with poorly controlled diabetes: Findings from a randomized controlled trial. Annals of Behavioral Medicine, 44, 207-215. 
Erickson, S. J., Gerstle, M., \& Feldstein, S. W. (2005). Brief interventions and motivational interviewing with children, adolescents, and their parents in pediatric health care settings. Archives of Pediatric Adolescent Medicine, 159, 1173-1180.

*Flattum, C., Friend, S., Story, M., \& Neumark-Sztainer, D. (2011). Evaluation of an individualized counseling approach as part of a multicomponent school-based program to prevent weight-related problems among adolescent girls. Journal of the American Dietetic Association, 111(8), 1218-1223.

*Freudenthal, J. J., \& Bowen, D. M. (2010). Motivational interviewing to decrease parental riskrelated behaviors for early childhood caries. Journal of Dental Hygiene, 84(1), 28-33.

*Halterman, J. S., Riekert, K., Bayer, A., Fagnano, M., Tremblay, P., Blaakman, S., \& Borrelli, B. (2011). A pilot study to enhance preventive asthma care among urban adolescents with asthma. Journal of Asthma, 48(5), 523-530.

*Halterman, J. S., Szilagyi, P. G., Fisher, S. G., Fagnano, M., Tremblay, P., Conn, K. M., ... Borrelli, B. (2011). Randomized controlled trial to improve care for urban children with asthma. Archives of Pediatric and Adolescent Medicine, 165(3), 262-268.

*Harrison, R., Benton, T., Everson-Stewart, S., \& Weinstein, P. (2007). Effect of motivational interviewing on rates of early childhood caries: A randomized trial. Pediatric Dentistry, 29(1), 16-22.

Heckman, C. J., Egleston, B. L., \& Hofmann, M. T. (2010). Efficacy of motivational interviewing for smoking cessation: A systematic review and meta-analysis. Tobacco Control, 19, 410-416.

Hettema, J. E., \& Hendricks, P. S. (2010). Motivational interviewing for smoking cessation: A meta-analytic review. Journal of Consulting and Clinical Psychology, 78(6), 868-884. 
Hettema, J., Steele, J., \& Miller, W.R. (2005). Motivational interviewing. Annual Review of Clinical Psychology, 1, 91-111.

*Ismail, A. I., Ondersma, S., Jedele, J. M. W., Little, R. J., \& Lepkowski, J. M. (2011).

Evaluation of a brief tailored motivational intervention to prevent early childhood caries. Community Dentistry and Oral Epidemiology, 39, 433-448.

Jensen, C. D., Cushing, C. C., Aylward, B. S., Craig, J. T, Sorell, D. M., \& Steele, R. G. (2011). Efficacy of motivational interviewing interventions for adolescent substance use behavior change: A meta-analytic review. Journal of Consulting and Clinical Psychology, 79(4), 433-440.

Kitzmann, K. M., Dalton, W. T., Stanley, C. M., Beech, B. M., Reeves, T. P., Buscemi, J., ... Midgett, E. L. (2010). Lifestyle interventions for youth who are overweight: A metaanalytic review. Health Psychology, 29(1), 91-101.

Knight, K. M., McGowan, L., Dickens, C., \& Bundy, C. (2006). A systematic review of motivational interviewing in physical health care settings. British Journal of Health Psychology, 11, 319-332.

Lundahl, B. W., Kunz, C., Brownell, C., Tollefson, D., \& Burke, B. L. (2010). A meta-analysis of motivational interviewing: Twenty-five years of empirical studies. Research on Social Work Practice, 20, 137.

*MacDonell, K., Brogan, K., Naar-King, S., Ellis, D., \& Marshall, S. (2012). A pilot study of motivational interviewing targeting weight-related behaviors in overweight or obese African American adoelscents. Journal of Adolescent Health, 50, 201-203. 
*Manarino, M. (2007). A motivational interviewing technique to improve calcium intake among adolescent girls. (Master's thesis). Retrieved from ProQuest Dissertations and Theses. (1451193)

Mehlenbeck, R. S., \& Wember, Y. M. (2008). Motivational interviewing and pediatric obesity. In E. Jelalian \& R. G. Steele (Eds.), Handbook of childhood and adolescent obesity (pp. 405-424). New York, NY: Springer.

Miller, W. R., \& Baca, L.M. (1983). Two-year follow-up of bibliotherapy and therapist directed controlled drinking training for problem drinking. Behavior Therapy, 14, 441-448.

Miller, W. R., \& Rollnick, S. (2002). Motivational Interviewing: Preparing people for change ( $2^{\text {nd }}$ ed.). New York: Guilford Press.

Miller, W. R., \& Rose, G. S. (2009). Toward a theory of motivational interviewing. American Psychologist, 64, 527-537.

*Naar-King, S., Outlaw, A., Green-Jones, M., Wright, K., \& Parsons, J. T. (2009). Motivational interviewing by peer outreach workers: A pilot randomized clinical trial to retain adolescents and young adults in HIV care. AIDS Care, 21(7), 868-873.

*Naar-King, S., Parsons, J. T., Murphy, D. A., Chen, X., Harris, R., \& Belzer, M. E. (2009). Improving health outcomes for youth living with the Human Immunodeficiency Virus. Archives of Pediatric and Adolescent Medicine, 163(12), 1092-1098.

*Naar-King, S., Wright, K., Parsons, J. T., Frey, M., Templin, T., Lam, P., \& Murphy, D. (2006). Healthy choices: Motivational enhancement therapy for health risk behaviors in HIVpositive youth. AIDS Education and Prevention, 18(1), 1-11.

Nemcek, M. A., \& Sabatier, R. (2003). State of evaluation: Community health workers. Public Health Nursing, 20(4), 260-270. 
*Neumark-Sztainer, D. R., Friend, S. E., Flattum, C. F., Hannan, P. J., Story, M. T., Bauer, K. W., ... Petrich, C. A. (2010). New moves- Preventing weight-related problems in adolescent girls. American Journal of Preventive Medicine, 39(5), 421-432.

*Obarzanek, E., Kimm, S. Y. S., Barton, B. A., Van Horn, L., Kwiterovich, P. O., SimonsMorton, D. G., ... Greenlick, M. R. (2001). Long-term safety and efficacy of a cholesterol-lowering diet in children with elevated low-density lipoprotein cholesterol: Seven-year results of the dietary intervention study in children (DISC). Pediatrics, 107(2), 256-264.

*Olson, A. L., Gaffney, C. A., Lee, P. W., \& Starr, P. (2008). Changing adolescent health behaviors: The healthy teens counseling approach. American Journal of Preventive Medicine, 35(5S), S359-S364.

Resnicow, K., Davis, R., \& Rollnick, S. (2006). Motivational interviewing for pediatric obesity: Conceptual issues and evidence review. Journal of the American Dietetic Association, 106, 2024-2033.

*Resnicow, K., Taylor, R., Baskin, M., \& McCarty, F. (2005). Results of Go Girls: A weight control program for overweight African-American adolescent females. Obesity Research, 13(10), 1739-1748.

*Riekert, K. A., Borrelli, B., Bilderback, A., \& Rand, C. S. (2011). The development of a motivational interviewing intervention to promote medication adherence among innercity, African-American adolescents with asthma. Patient Education and Counseling, 82, $117-122$.

*Robertson, A. R., St. Lawrence, J., Morse, D. T., Baird-Thomas, C., Liew, H., \& Gresham, K. (2011). The healthy teen girls project: Comparison of health education and STD risk 
reduction intervention for incarcerated adolescent females. Health Education and Behavior, 38, 241-250.

Rubak, S., Sandbaek, A., Lauritzen, T., \& Christensen, B. (2005). Motivational interviewing: A systematic review and meta-analysis. British Journal of General Practice, 55, 305-312.

*Schmiege, S. J., Broaddus, M. R., Levin, M., \& Bryan, A. D. (2009). Randomized trial of group interventions to reduce HIV/STD risk and change theoretical mediators among detained adolescents. Journal of Consulting and Clinical Psychology, 77(1), 38-50.

*Schwartz, R. P., Hamre, R., Dietz, W. H., Wasserman, R. C., Slora, E. J., Myers, E. F., ... Resnicow, K. A. (2007). Office-based motivational interviewing to prevent childhood obesity. Archives of Pediatric \& Adolescent Medicine, 161, 495-501.

*Seid, M., D’Amico, E. J., Varni, J. W., Munafo, J. K., Britto, M. T., Kercsmar, C. M., ... Darbie, L. (2012). The in vivo adherence intervention for at risk adolescents with asthma: Report of a randomized pilot trial. Journal of Pediatric Psychology, 37(4), 390-403.

Sindelar, H. A., Abrantes, A. M., Hart, C., Lewander, W., \& Spirito, A. (2004). Motivational interviewing in pediatric practice. Current Problems in Pediatric Adolescent Health Care, 34, 322-339.

Stacciarini, J. R., Rosa, A., Ortiz, M., Munari, D. B., Uicab, G., \& Balam, M. (2012). Promotoras in mental health: A review of English, Spanish, and Portuguese literature. Family Community Health, 35(2), 92-102.

Suarez, M., \& Mullins, S. (2008). Motivational interviewing and pediatric health behavior interventions. Journal of Developmental and Behavioral Pediatrics, 29, 417-428. 
Tait, R. J., \& Hulse, G. K. (2003). A systematic review of the effectiveness of brief interventions with substance using adolescents by type of drug. Drug and Alcohol Review, 22, 337346.

*Taveras, E. M., Blackburn, K., Gillman, M. W., Haines, J., McDonald, J., Price, S., \& Oken, E. (2011). First steps for mommy and me: A pilot intervention to improve nutrition and physical activity behaviors of postpartum mothers and their infants. Maternal and Child Health Journal, 15, 1217-1227.

*Taveras, E. M., Gortmaker, S. L., Hohman, K. H., Horan, C. M., Kleinman, K. P., Mitchell, K., ... Gillman, M. W. (2011). Randomized controlled trial to improve primary care to prevent and manage childhood obesity. Archives of Pediatric and Adolescent Medicine, 165(8), 714-722.

Tevyaw, T. O., \& Monti, P. M. (2004). Motivational enhancement and other brief interventions for adolescent substance abuse: Foundations, applications, and evaluations. Addiction, 99, $2,63-75$.

U.S. Department of Health and Human Services, Health Resources and Services Administration, Bureau of Health Professions. (2007). Community health worker national workforce study. Retrieved from http://minorityhealth.hhs.gov/templates/content.aspx?lvl=2\&lvlid=207\&ID=8930

Vasilaki, E., Hosier, S., \& Cox, W. (2006). The efficacy of motivational interviewing as a brief intervention for excessive drinking: A meta-analytic review. Alcohol and Alcoholism, 41, $328-335$. 
*Viner, R. M., Christie, D., Taylor, V., \& Hey, S. (2003). Motivational/solution-focused intervention improves $\mathrm{HbA}_{1 \mathrm{C}}$ in adolescents with Type 1 Diabetes: A pilot study. Diabetes Medicine, 20, 739-742.

*Wang, Y., Nakronezny, P. A., Stewart, S. M., Edwards, D., Mackenzie, M., \& White, P. C. (2010). A randomized controlled trial comparing motivational interviewing in education to structured diabetes education in teens with type 1 diabetes. Diabetes Care, 33(8), 1741-1743.

Wachtel, T., \& Staniford, M. (2010). The effectiveness of brief interventions in the clinical setting in reducing alcohol misuse and binge drinking in adolescents: A critical review of the literature. Journal of Clinical Nursing, 19, 605-620.

*Weinstein, P., Harrison, R., \& Benton, T. (2006). Motivating mothers to prevent caries. Journal of the American Dental Association, 137, 789-793.

*Wilhelm, S. L., Stephans, M. B. F., Hertzog, M., Rodehorst, T. K. C., \& Gardner, P. (2006). Motivational interviewing to promote sustained breastfeeding. Journal of Obstetric, Gynecologic, \& Neonatal Nursing, 35(3), 340-348.

*Wilson, D. K., Friend, R., Teasley, N., Green, S., Reaves, I. L., \& Sica, D. A. (2002).Motivational versus social cognitive interventions for promoting fruit and vegetable intake and physical activity in African American adolescents. Annals of Behavioral Medicine, 24(4), 310-319.

Wisdom, J. P., Cavaleri, M., Gogel, L., \& Nacht, M. (2011). Barriers and facilitators to adolescent drug treatment: Youth, family, and staff reports. Addiction Research and Theory, 19(2), 179-188. 


\section{Appendix}

Measurement of Study Quality: Study Rigor Rating System (adapted from Lundahl et al., 2010):

1) Demographic variable points (1 point for each of the following):

- Reported on at least three or more demographic indicators of the sample

- Collected data at a follow-up period beyond immediate completion of the study

- Collected data at more than one site

- Included more than one site, reported data from all dependent variables they assessed

- Utilized coders who were "blind" to participants' group assignment

- Utilized objective measurement tools (e.g., records, physiological indicators) instead of relying solely on client self-report

- Utilized a manual to direct training or standardized delivery

- Reported on drop- outs

- Included more than 20 participants in the intervention and comparison groups.

2) Effect size calculation points (2 points maximum):

- 2 points if the data used to calculate effect sizes came from means, standard deviations, and/or numbers of participants (percentages)

- 1 point if an exact statistic was used (e.g., $t$ test)

- No point if effect sizes were derived from $p$ values

3) Data reporter points (2 points maximum):

- 2 points if measurement of outcomes came from at least two sources (e.g., participant and collateral source)

- 1 point if collateral only, and no point if participant only.

4) Fidelity measurement points ( 2 points maximum): 
- Studies earned 2 points if fidelity was assessed and considered high

- 1 point if fidelity was assessed but not scored

- No point if fidelity was not measured.

5) Randomization points (3 points maximum):

- 3 points if true randomization was used

- 2 points if matched groups were used (adapted to also include block randomization, e.g., by doctor's office or school)

- 1 point if the groups were tested for pretreatment equivalence

- No point if groups were not equivalent or equivalence could not be determined 